\title{
Blood-born tissue factor in cardiovascular disease: where are we now?
}

\author{
Vincenzo Toschi
}

Received: 1 August 2010/Accepted: 31 August 2010/Published online: 18 September 2010

(C) SIMI 2010

\section{Atherosclerotic plaque thrombogenicity: the role of tissue factor}

Over the last decade, many clinical, pathological and laboratory data have been obtained indicating that, in the majority of cases, acute coronary thrombosis occurs upon rupture of the thin fibrous cap of a vulnerable atherosclerotic plaques, a result of a series of immuno-inflammatory processes. The inner content of the plaque becomes then exposed to the circulating blood leading to the deposition of thrombotic material into the lumen [1]. Our data, as well as that acquired by others, demonstrate that one of the major determinants of the thrombogenicity of the disrupted atheroma is tissue factor (TF), which triggers the activation of coagulation by binding to factor VII/VIIa. TF is particularly abundant in the lipid-rich necrotic core of the lesion, which is therefore the most thrombogenic component of atherosclerotic plaque [2]. The binding of TF to factor VIIa causes thrombin generation, which in turn induces platelet activation and fibrin formation leading to a partial or total thrombotic occlusion of the artery, and to the occurrence of an acute coronary syndrome (ACS) [1]. We also have demonstrated that the activation of the coagulation cascade within the coronary vessel at sites of atherosclerotic plaque rupture is largely TF-dependent, since a significant reduction in both platelet and fibrin deposition can be obtained by local pretreatment of atherosclerotic lesions with the recombinant analog of the physiologic tissue factor pathway inhibitor (rTFPI) or with

\section{Toschi $(\square)$}

Department of Hematology and Blood Transfusion, Thrombosis Center, A.O. Ospedale San Carlo Borromeo, Via Pio II, 3, 20153 Milan, Italy

e-mail: vincenzo.toschi1@alice.it a specific anti-TF antibody [3]. Instabilization and rupture of the plaque are mainly due to inflammatory processes that take place in the vessel wall. Experimental studies demonstrate that inflammatory cells such as macrophages, macrophage-derived lipid laden 'foam cells', and T lymphocytes are major constituents of plaques, and infiltrate areas of superficial erosion and the shoulder region of the coronary lesions where the fibrous cap usually breaks [4]. Macrophages and 'foam cells' are also the major source of TF within the lesions, and therefore this protein can be considered a product of the inflammatory processes of the atheroma [1]. The interplay between these cells results in the production and local secretion of several inflammatory cytokines and chemokines like interleukin (IL)-1, interferon- $\gamma$ and TNF- $\alpha$, which, in turn, lead to the release of metalloproteinases (MMPs), particularly of MMP-9, which degrade extracellular matrix and particularly collagen, thus inducing plaque weakening and fracture $[1,5,6]$.

\section{The blood hypercoagulability}

It has been shown that, besides local factors, systemic prothrombotic mediators may contribute to coronary thrombosis, thus suggesting that atherothrombosis is a complex and multifactorial process. Prospective studies have shown that some hemostatic biomarkers may predict future cardiovascular events, both in apparently healthy subjects and in patients with ACS. Specifically, Merlini et al. show a persistent activation of coagulation in patients who experience an ACS, as suggested by increased plasma levels of prothrombin fragment $\mathrm{F} 1+2$ indicating ongoing thrombin generation, which is still detectable several months after the acute event. The same investigators also show that plasma F1 +2 and fibrinopeptide A, measured on hospital 
admission in ACS patients, are significantly higher in those patients who subsequently develop an adverse outcome such as cardiac death or (re)infarction, than in those who have an uneventful clinical course over a 72 -h observation period [7]. Thus, this indicates that the extent of systemic activation of coagulation with increased thrombin generation and activity can have an unfavorable prognosis. Moreover, recent studies, mainly by Nemerson's group, indicate that plaque-related TF exposure alone may not be enough to sustain total arterial occlusion, since, after TF exposure, the formation of a $1 \mathrm{~mm}$ thrombus may require up to $16 \mathrm{~h}$. In fact, the time required to activate factor $\mathrm{X}$, which plays a crucial role in thrombin generation, to diffuse $1 \mathrm{~mm}$ from the TF-rich surface of the atheroma to the apex of fibrin growing thrombus is $4 \mathrm{~h}$, and becomes 3.6 months when platelet deposition occurs [8]. The authors conclude that as complete thrombotic occlusion is observed within $10 \mathrm{~min}$, an alternatively source of procoagulant activity, able to localize coagulation factors to the outer surface of growing thrombi, appears essential for rapid thrombus growth. This observation led to the hypothesis of circulating (blood-born) TF.

\section{Blood thrombogenicity: the role of circulating tissue factor}

In this issue of Internal and Emergency Medicine, Cimmino and co-workers provide an extensive review of blood-born tissue factor as a possible additional candidate to induce a pro-coagulant systemic state in ACS, and to contribute to a local arterial thrombus growth [9]. Experimental studies indicate that circulating TF may be important in sustaining atherothrombotic phenomena in addition to locally active TF present in the plaque. Blood-borne TF, released by leukocytes, is essential for thrombus propagation after its initial formation on the vascular surface [10]. Further studies suggest that platelets might also be a potential source of circulating TF. Our group has shown that immunoreactive and functionally active TF is found on platelet membrane, and its expression may be increased after stimulation of these cells by agonists such as ADP and thrombin [11]. We also demonstrate that de novo synthesis of TF by platelets may take place since TF mRNA can be found in these cells [12]. It is therefore conceivable that platelet associated, functionally active, TF might be delivered by these cells at the site of thrombus formation thus contributing to its growth. However, other potential sources of circulating TF have been reported and summarized in a recent review [13]. These are monocytes and macrophages, granulocytes and microparticles, mostly of platelet origin, but the TF is also released by red blood cells, granulocytes, monocytes, lymphocytes, endothelial cell and smooth muscle cells. Finally, a soluble form of circulating human $\mathrm{TF}$, generated by alternative splicing of the primary RNA transcript has been described [14]. This soluble TF fraction, corresponding to approximately $10-30 \%$ of TF detectable in human plasma, contains most of the extracellular domain of TF, but lacks a transmembrane domain, therefore missing membrane anchorage.

\section{Is circulating TF functionally active?}

Studies demonstrate that only a small amount of bloodborn TF exerts pro-thrombotic activity. The inactive form of circulating TF is 'encrypted' and 'decription' of the molecule is essential for its activity.

\section{Clinical significance of blood-born TF in patients with $\mathrm{ACS}$}

Several atherosclerotic risk factors such as hypertension, dislipidemia, and diabetes mellitus are associated with elevated blood-borne TF antigen and activity, as well as with increased TF expression in atherosclerotic plaques. Pharmacological treatment of these conditions reduces TF over-expression [13]. Brambilla [15] recently report that platelet TF is higher in patients with ACS when compared with normal subjects and to patients with stable angina, and that TF over-expression in ACS patients is associated with an increase in platelet TF mRNA. Taken together, these findings seem to suggest that circulating TF may play an active role in the pathogenesis of ACS, and that therefore it could be a potential target for therapy.

Conflict of interest None.

\section{References}

1. Libby P (2008) The molecular mechanisms of the thrombotic complications of atherosclerosis. J Intern Med 263:517-527

2. Toschi V, Gallo R, Lettino M, Fallon JT, Gertz SD, FernandezOrtiz A, Chesebro JH, Badimon L, Nemerson Y, Fuster V, Badimon JJ (1997) Tissue factor modulates the thrombogenicity of human atherosclerotic plaques. Circulation 95:594-599

3. Fuster V, Moreno PR, Fayad ZA, Corti R, Badimon JJ (2005) Atherothrombosis and high risk plaque. J Am Coll Cardiol 46:937-954

4. Badimon JJ, Lettino M, Toschi V, Fuster V, Berrozpe M, Chesebro JH, Badimon L (1999) Local inhibition of tissue factor reduces the thrombogenicity of disrupted human atherosclerotic plaques: effects of tissue factor pathway inhibitor on plaque thrombogenicity under flow conditions. Circulation 99:1780-1787

5. Virmani R, Burke AP, Farb A, Kolodgie FD (2006) Pathology of the vulnerable plaque. J Am Coll Cardiol 47(8 Suppl):C13-C18

6. Hansson GK (2005) Inflammation, atherosclerosis and coronary artery disease. N Engl J Med 352:1686-1695 
7. Deguchi JO, Aikawa M, Tung C-H, Aikawa E, Kim D-E, Ntziachristos V, Weissleder R, Libby P (2006) Inflammation in atherosclerosis. Visualizing matrix metalloproteinase action in macrophages in vivo. Circulation 114:55-62

8. Merlini PA, Ardissino D, Bauer KA, Oltrona L, Pezzano A, Bottasso B, Rosemberg RD, Mannucci PM (1997) Persistent thrombin generation during heparin therapy in patients with acute coronary syndromes. Arterioscler Thromb Vasc Biol $17: 1325-1330$

9. Hathcock JJ, Nemerson Y (2004) Platelet deposition inhibits tissue factor activity: in vitro clots are impermeable to factor Xa. Blood 104:123-127

10. Cimmino G, Golino P, Badimon JJ (2010) Pathophysiological role of blood-born tissue factor: should the old paradigm be revisited? Intern Emerg Med. doi:10.1007/s11739-010-0423-4

11. Giesen PLA, Rauch U, Bohrman B, Kling D, Roque M, Fallon JT, Badimon JJ, Himber J, Riederer MA, Nemerson Y (1999)
Blood-born tissue factor: another view of thrombosis. Proc Natl Acad Sci USA 96:2311-2315

12. Camera M, Frigerio M, Toschi V, Brambilla M, Rossi F, Cottell DC, Maderna P, Parolari A, Bonzi R, De Vincenti O, Tremoli E (2003) Platelet activation induces cell-surface immunoreactive tissue factor expression, which is modulated differently by antiplatelet drugs. Arterioscler Thromb Vasc Biol 23:1690-1696

13. Breitenstein A, Tanner FC, Lüscher TF (2010) Tissue factor in cardiovascular disease. Circ J 74:3-12

14. Bogdanov VY, Balasubramanian V, Hathcock J, Vele O, Lieb M, Nemerson Y (2003) Alternatively spliced human tissue factor: a circulating, soluble, thrombogenic protein. Nat Med 9:458-462

15. Brambilla M, Camera M, Colnago D, Marenzi G, De Metrio M, Giesen P, Balduini A, Veglia F, Gertow K, Biglioli P, Tremoli E (2008) Tissue factor in patients with acute coronary syndromes. Expression in platelets, leukocytes, and platelet-leukocytes aggregates. Arterioscler Thromb Vasc Biol 28:947-953 